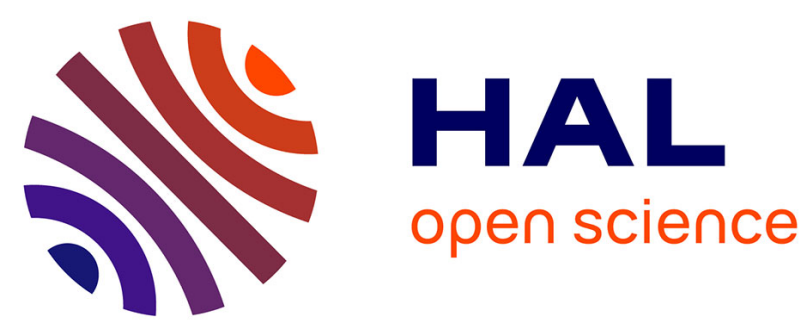

\title{
Dependency of the Dimethyldihydropyrene Photochromic Properties on the Number of Pyridinium Electron-Withdrawing Groups
}

Elise Lognon, Marie-Catherine Heitz, Assil Bakkar, Saioa Cobo, Frédérique Loiseau, Eric Saint-aman, Guy Royal, Martial Boggio-Pasqua

\section{To cite this version:}

Elise Lognon, Marie-Catherine Heitz, Assil Bakkar, Saioa Cobo, Frédérique Loiseau, et al.. Dependency of the Dimethyldihydropyrene Photochromic Properties on the Number of Pyridinium ElectronWithdrawing Groups. ChemPhysChem, 2020, 21 (14), pp.1571-1577. 10.1002/cphc.202000304 . hal03011523

\section{HAL Id: hal-03011523 \\ https://hal.science/hal-03011523}

Submitted on 30 Nov 2020

HAL is a multi-disciplinary open access archive for the deposit and dissemination of scientific research documents, whether they are published or not. The documents may come from teaching and research institutions in France or abroad, or from public or private research centers.
L'archive ouverte pluridisciplinaire $\mathbf{H A L}$, est destinée au dépôt et à la diffusion de documents scientifiques de niveau recherche, publiés ou non, émanant des établissements d'enseignement et de recherche français ou étrangers, des laboratoires publics ou privés. 


\title{
Dependency of the Dimethyldihydropyrene Photochromic Properties on the Number of Pyridinium Electron-Withdrawing Groups
}

\author{
Elise Lognon, ${ }^{[\mathrm{a}]}$ Marie-Catherine Heitz, ${ }^{[\mathrm{a}]}$ Assil Bakkar, ${ }^{[\mathrm{b}]}$ Saioa Cobo, ${ }^{[\mathrm{b}]}$ Frédérique Loiseau, ${ }^{[\mathrm{b}]}$ Eric \\ Saint-Aman, ${ }^{[b]}$ Guy Royal, ${ }^{[b]}$ and Martial Boggio-Pasqua ${ }^{*[a]}$
}

In honor of Olivier Poizat
[a] E. Lognon, Dr. M.-C. Heitz, Dr. M. Boggio-Pasqua LCPQ UMR 5626
CNRS et Université Toulouse III - Paul Sabatier
118 route de Narbonne, 31062 Toulouse, France
E-mail: martial.boggio@irsamc.ups-tlse.fr
[b] Dr. A. Bakkar, Dr. S. Cobo, Prof. F. Loiseau, Prof. E. Saint-Aman, Prof. G. Royal
Univ. Grenoble Alpes
CNRS UMR 5250, DCM
F-38000 Grenoble, France

Supporting information for this article is given via a link at the end of the document.

\begin{abstract}
Photochromic dimethyldihydropyrenes substituted with electron-withdrawing pyridinium groups have shown an increase of photo-induced ring-opening efficiency and a light sensitivity that is red shifted relative to the unsubstituted compound. However, a recently synthesized tetrapyridinium derivative showed a considerable decrease of the photo-isomerization quantum yield relative to the monopyridinium and bispyridinium derivatives. We provide a rationale for this unexpected photochemical behavior based on the comparative theoretical investigations of the relevant excited states of these systems. In particular, we found that the nature and order of the lowest two excited states depend on the number of pyridinium groups and on the symmetry of the system. While the lowest $S_{1}$ excited state is photo-active in the monopyridinium and bispyridinium derivatives, the photo-isomerizing state is $S_{2}$ in the reference unsubstituted compound and both $S_{1}$ and $S_{2}$ lead to isomerization in the tetrapyridinium derivative, albeit with a low efficiency. In the latter derivative, the photo-isomerization is hindered by the particular $S_{1} / S_{2}$ conical intersection topology.
\end{abstract}

\section{Introduction}

Besides being a clean and inexhaustible energy source, light is now envisioned as a powerful way to control the physical properties of materials, for applications in micro and nanotechnologies. Among them, molecular systems exhibiting switchable optical properties, emission and excitation fluorescence and electronic communication by light are among the most promising topics in the field of nanosciences. ${ }^{[1]}$

During the last decade, the dimethyldihydropyrene (DHP) cyclophanediene (CPD) photochromic couple has gained an increasing interest because of its original behavior. ${ }^{[2]}$ In particular it belongs to the class of negative photochromic systems. Indeed, this system switches by irradiation with visible light between a highly conjugated closed-ring isomer (DHP) and a less aromatic open-ring counterpart (CPD). The reverse process from the CPD to the DHP isomer is achieved by UV-irradiation or heating (Scheme 1). ${ }^{[3]}$

The chemical modification of the DHP photochromic core by one or two electron-withdrawing pyridinium substituents $\left(\mathbf{2}^{+}\right.$and $\mathbf{3 c}^{2+}$; Scheme 1) has recently been studied and the photo-isomerization quantum yield has been improved by at least one order of magnitude relative to the unsubstituted reference compound $1_{\mathrm{c}} \cdot{ }^{\left[{ }^{[4]}\right.}$ Indeed, the ring-opening quantum yield increases from $0.006^{\left[{ }^{[5]}\right.}$ in $1 \mathrm{c}$ to 0.040 and $0.042^{[4]}$ in $2 \mathrm{c}^{+}$and $3 \mathrm{c}^{2+}$, respectively. This improved photoswitching behavior was rationalized by theoretical calculations: in the reference compound, the photo-isomerization can be triggered by blue light irradiation $(\lambda>480 \mathrm{~nm}),{ }^{[5]}$ populating a $S_{2}$ zwitterionic excited state dominated by a HOMO $\rightarrow$ LUMO transition. ${ }^{[6]}$ This state is photo-active (i.e., state that leads to photo-isomerization) but is largely depopulated by internal conversion because of the presence of a lower $S_{1}$ covalent excited state, which is unreactive (i.e., does not lead to isomerization). ${ }^{[6]}$ In the mono and bispyridinium substituted DHPs, the $S_{1}$ and $S_{2}$ excited states gain charge transfer characters and the $S_{1}$ excited state becomes the photo-active state upon red light irradiation $(\lambda>630 \mathrm{~nm})$ with an improved efficiency. ${ }^{[4]}$

The tetrapyridinium DHP derivative $\left(\mathbf{4}^{\mathbf{4}^{+*}}\right.$; Scheme 1$)$ was later synthesized and showed very different electrochemical and photochemical behaviors compared to its bispyridinium counterpart. ${ }^{[7]}$ On the one hand, $\mathbf{4 c}^{{ }^{4+}}$ undergoes an efficient ringopening isomerization leading to 4o upon electrochemical reduction, whereas $3_{c}{ }^{2+}$ does not produce its corresponding openring isomer 3 o when reduced. Thus, the use of pyridinium redoxactive units can provide a strategy for the electrochemically triggered switching processes of DHPs. On the other hand, the photo-isomerization quantum yield of $4 c^{4+}$ was measured with a 300 -fold decrease compared to $3 \mathrm{c}^{2+}$ (and is also lower than the parent $\mathbf{1}_{\mathrm{c}}$ ), which is a surprising and yet unexplained observation. 


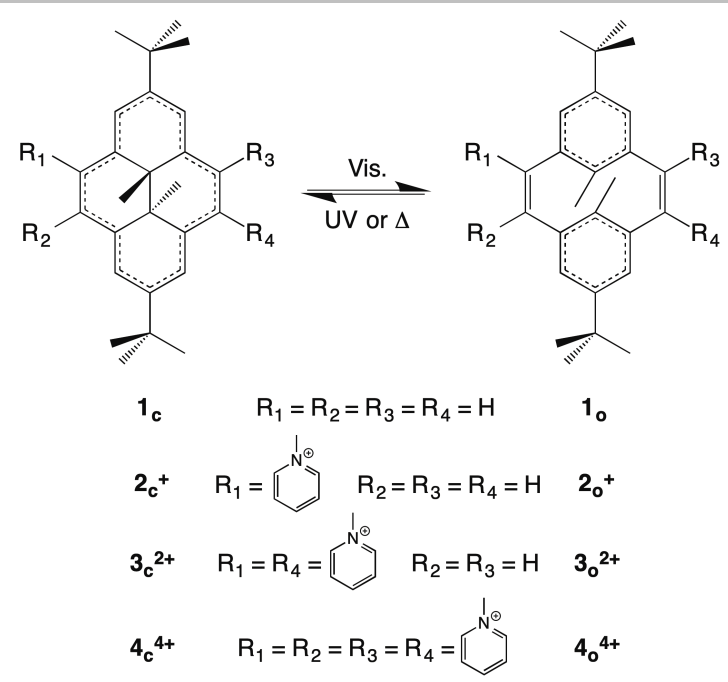

Scheme 1. DHP (closed form) - CPD (open form) isomerization and various pyridinium-appended systems studied.

In this study, in order to provide a rationale for the unexpectedly poor efficiency of the tetrapyridinium DHP derivative $\mathbf{4 c}^{\mathbf{4}^{+}}$, we investigate its photoswitching behavior using theoretical calculations based on density functional theory (DFT) and timedependent DFT (TD-DFT) and we compare our results with those of the mono $2_{\mathrm{c}}{ }^{+}$and bispyridinium $3_{\mathrm{c}}{ }^{2+}$ derivatives. First, the computed ground-state structure of $\mathbf{4 c}^{4+}$ is compared with the experimental $\mathrm{X}$-ray data and the thermal isomerization pathway is investigated through the characterization of the biradicaloid transition state between the closed- and open-ring isomers and its associated energy barrier. Second, we investigate the potential energy landscape of the first two excited states $S_{1}$ and $S_{2}$ along the initial photo-isomerization pathway and we show how it differs from that of $2^{+}$and $3 c^{2+}$. Finally, we propose a possible explanation for the change of behavior observed in $4 c^{4+}$ based on steric and electronic factors.

\section{Results and Discussion}

Ground-state properties. Single crystals of $4 \mathrm{c}^{4+}$ suitable for Xray analysis have been obtained by slow diffusion of diethyl ether into an acetonitrile solution of the compound. $\mathbf{4 c}^{{ }^{4+}}$ crystallizes in the space group $\mathrm{C} 2 / \mathrm{c}$ (monoclinic system). The X-ray structure (Figure 1 and Table $S 1$ in Supporting Information) reveals that the length of the central $C-C$ bond is $1.522 \AA$, which is similar to values observed for other DHP derivatives. The distance is also in good agreement with the computed value of $1.545 \AA$ for the isolated system using DFT (Table 1). In this experimental structure, the four pyridinium units adopt a twisted arrangement, with dihedral angles with respect to the main DHP plane of $64^{\circ}$ for two pyridinium and $76^{\circ}$ for the other two. The DFT structure of the isolated model system (see Computational Section and Table S2 in Supporting Information) belongs to the $C_{2 h}$ point group and the torsion angle with respect to the DHP core is $64^{\circ}$ for the four pyridinium groups. This torsion is more pronounced than that of the corresponding monopyridinium- and bispyridinium-appended DHP for which torsion angles of $34^{\circ}$ and $42^{\circ}$ were computed, respectively (Table 1). ${ }^{[4]}$ Note also that the introduction of pyridinium groups slightly breaks the planarity of the DHP core by a few degrees compared to the reference compound $\mathbf{1}_{\mathrm{c}}$ (Table 1).

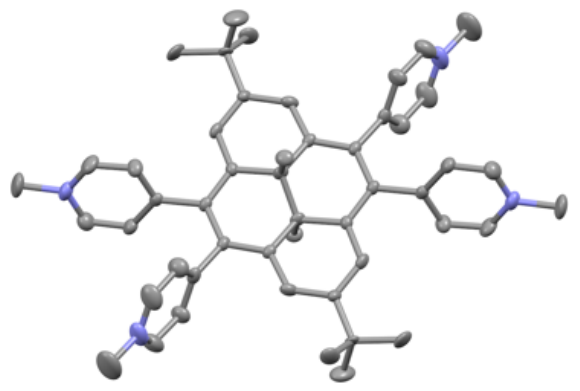

Figure 1. Molecular structure of $\mathbf{4 c}^{4+}$. Ellipsoids are set at $50 \%$ probability; hydrogen atoms and anions have been omitted for clarity.

Table 1. Geometrical parameters ${ }^{[a]}$ characterizing the ground-state structures of the studied DHP derivatives.

\begin{tabular}{llll}
\hline DHPs & $\mathrm{q}(\AA)$ & $\phi\left(^{\circ}\right)$ & $\tau\left({ }^{\circ}\right)$ \\
\hline $\mathbf{1} \mathbf{c}$ & 1.543 & 2.4 & - \\
$\mathbf{2}^{+}$ & 1.547 & 6.2 & 34 \\
$\mathbf{3 c}^{2+}$ & 1.549 & 7.9 & 42 \\
$\mathbf{4 c}^{4+}$ & 1.545 & 7.7 & 64 \\
\hline
\end{tabular}

[a] $q$ is the transannular bond distance, $\phi$ is an average dihedral angle measuring the DHP planarity and $\tau$ is the torsion angle of the pyridinium groups with respect to the DHP core. Taken from Ref. [12] for 1c and from Ref. [4] for $3 c^{2+}$

DFT calculations confirm that the DHP isomers are more stable than their respective CPD forms by about $20 \mathrm{kcal} \cdot \mathrm{mol}^{-1}$ (Table 2). Interestingly, all CPDs exhibit similar thermal isomerization barriers of about 18 to $20 \mathrm{kcal} \cdot \mathrm{mol}^{-1}$, values in agreement with those reported in the literature for other related DHPs. ${ }^{\left[{ }^{[8,9]}\right.}$ We can thus predict that the thermal stability of all these CPDs is rather similar. Note that the transition states involved have a strong biradical nature, as indicated by their heavily spin-contaminated wave function $\left(<S^{2}>\approx 1\right)$. This is consistent with previous studies of other DHP/CPD couples. ${ }^{[10]}$

Table 2. Computed relative energies between closed- and open-ring isomers and potential energy barriers for the thermal cyclization process.

\begin{tabular}{lll}
\hline Systems & $\Delta \mathrm{E}_{\mathrm{DHP} / \mathrm{CPD}}(\mathrm{kcal} / \mathrm{mol})$ & $\Delta \mathrm{E}_{\mathrm{CPD} \rightarrow \mathrm{DHP}}^{\ddagger}(\mathrm{kcal} / \mathrm{mol})$ \\
\hline $\mathbf{1}$ & 22.1 & 20.0 \\
$\mathbf{2}^{+}$ & 20.6 & 18.9 \\
$\mathbf{3}^{2+}$ & 17.1 & 18.9 \\
$\mathbf{4}^{4+}$ & 20.5 & 17.9 \\
\hline
\end{tabular}

Excited-state properties. The absorption spectrum of $4^{4+}$ recorded in acetonitrile at $3 \cdot 10^{-5} \mathrm{M}$ (Figure 2 ) shows four broad absorption bands at $672,496,398$ and $334 \mathrm{~nm}$. Based on TDDFT calculations (Table 3 and Figure $\mathrm{S} 1$ in Supporting Information), these bands can be attributed to $\pi-\pi^{*}$ transitions 
localized mainly within the DHP core but with some charge transfer character towards the electron-withdrawing pyridinium groups, as shown by the natural transition orbitals ${ }^{[11]}$ involved in some of these electronic transitions.

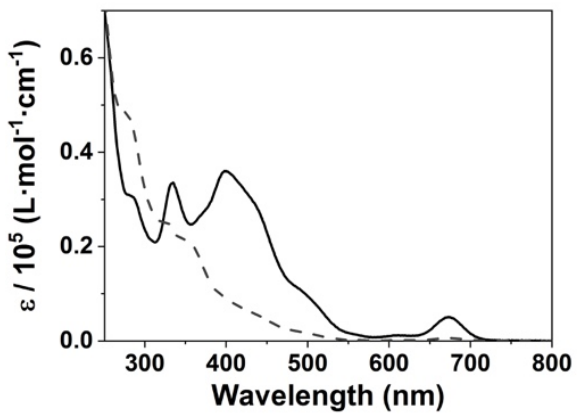

Figure 2. UV-Vis absorption spectra of $4^{4+}$ (full line) and $4^{4+}$ (dashed line) recorded in acetonitrile at $3 \cdot 10^{-5} \mathrm{M}$.

Table 3. UV/Vis absorption spectral data and TD-DFT results of $\mathbf{4}^{\mathbf{4}^{4+}}$ in acetonitrile.

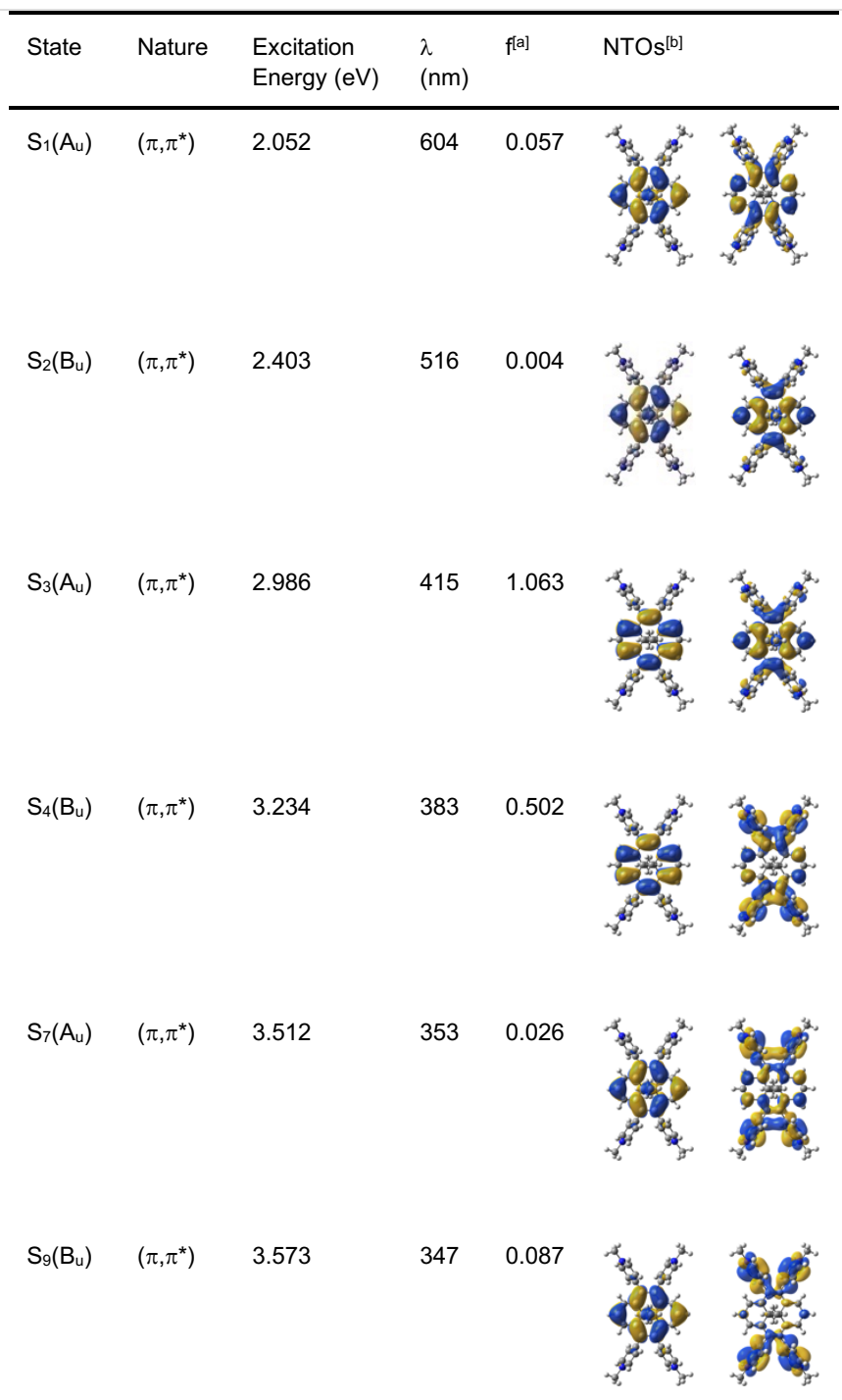

\section{$\mathrm{S}_{12}\left(\mathrm{~B}_{\mathrm{u}}\right) \quad\left(\pi, \pi^{*}\right) \quad 4.002 \quad 310 \quad 0.431$

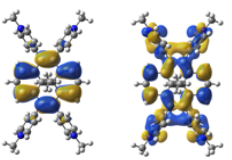

[a] Oscillator strength. [b] Main pair of natural transition orbitals.

Upon visible light irradiation ( $\lambda>630 \mathrm{~nm}$ or $\lambda>480 \mathrm{~nm}$ ), $\mathbf{4 c}^{4+}$ can be converted into its open-ring isomer $4_{o}{ }^{4+}$ with a low quantum yield. The C-C central bond opening and the concomitant loss of aromaticity are revealed by a decrease of the absorption bands in the visible region and the emergence of a new set of bands in the UV-region (Figure 2). As published previously, ${ }^{[4]}$ the introduction of one or two pyridinium units (in trans position of the DHP core; Scheme 1) enhances the photo-induced ring-opening efficiency compared to the reference $1_{c}$ system by one order of magnitude. In addition, the photo-isomerization in $2 \mathrm{c}^{+}$and $3 \mathrm{c}^{2+}$ can be triggered by exciting their $S_{1}$ state with red light, ${ }^{[4]}$ whereas $\mathbf{1 c}_{\mathrm{c}}$ needs to be excited in its $S_{2}$ state with blue light (no isomerization from $\left.S_{1}\right){ }^{[5]}$ Quantum chemical calculations brought a simple rationalization for this behavior: in the reference compound $\mathbf{1}_{c}$, the $\mathrm{DHP} \rightarrow \mathrm{CPD}$ photo-isomerization is largely inefficient because of the depopulation of the photo-active $S_{2}\left(\pi, \pi^{*}\right)$ zwitterionic $\left(\mathrm{L}_{a}\right)$ state by the lower $S_{1}\left(\pi, \pi^{*}\right)$ covalent $\left(L_{b}\right)$ state. While vibrational relaxation in the $S_{2}\left(\pi, \pi^{*}\right)$ state leads to a CPD precursor (denoted $\mathrm{CPD}^{*}$ herein) characterized by an elongated transannular $\mathrm{C}-\mathrm{C}$ bond and a loss of planarity of the DHP core relative to the ground-state structure, the $S_{1}\left(\pi, \pi^{*}\right)$ relaxes to an excited-state minimum (denoted $\mathrm{DHP}^{*}$ ) with a similar structure as ground-state DHP (see Table 4). From DHP*, the system deactivates back to the initial ground-state DHP either radiatively or non-radiatively. Thus, the $S_{1}\left(\pi, \pi^{*}\right) L_{b}$ state does not lead to CDP isomerization. ${ }^{[6,12,13]}$ In the mono $2 \mathrm{c}^{+}$and bispyridinium $3 \mathrm{c}^{2+} \mathrm{DHP}$ compounds, the $S_{1}$ and $S_{2}$ electronic excited states gain a partial charge transfer character from the DHP core to the electronwithdrawing pyridinium units. Furthermore, the $S_{1}$ state becomes the photo-active state, as this state leads directly to $\mathrm{CPD}^{*}$ by vibrational relaxation on $S_{1}$. No population quenching by a lower excited state is present and irradiation at low energy (red light) can be used to trigger the DHP $\rightarrow$ CPD photo-isomerization. ${ }^{[4,8,9]}$ Note that $\mathrm{CPD}^{*}$ is only an excited-state intermediate towards isomerization and that further vibronic couplings and nonadiabatic processes are involved in the nonradiative decay to $\mathrm{S}_{0}$ on the way to the CPD isomer. ${ }^{[6]}$

Table 4. Main geometrical parameters ${ }^{[a]}$ characterizing the minima on the $\mathrm{S}_{0}, \mathrm{~S}_{1}$ and $S_{2}$ potential energy surface of the studied DHPs.

\begin{tabular}{|c|c|c|c|c|c|c|c|c|}
\hline & $1_{c}$ & & $2 \mathrm{c}^{+}$ & & $3 c^{2+}$ & & $4_{c}^{4+}$ & \\
\hline & $q(\AA)$ & $\phi\left({ }^{\circ}\right)$ & $q(\AA)$ & $\phi\left({ }^{\circ}\right)$ & $\mathrm{q}(\AA)$ & $\phi\left({ }^{\circ}\right)$ & $q(\AA)$ & $\phi\left(^{\circ}\right)$ \\
\hline $\mathrm{S}_{0}{ }^{\min }$ & 1.543 & 2.4 & 1.547 & 6.2 & 1.549 & 7.9 & 1.545 & 7.7 \\
\hline $\mathrm{S}_{1}{ }^{\min }$ & 1.552 & 4.1 & 1.555 & 9.9 & 1.568 & 14.4 & 1.554 & 11.3 \\
\hline $\mathrm{S}_{2}{ }^{\min }$ & 1.608 & 12.3 & 1.552 & 7.6 & 1.558 & 10.8 & $1.613^{[b]}$ & $21.1^{[\mathrm{b}]}$ \\
\hline
\end{tabular}

[a] $\mathrm{q}$ is the transannular bond distance and $\phi$ is an average dihedral angle measuring the DHP planarity. Taken from Ref. [12] for $1_{c}$ and from Ref. [4] for $3^{2}{ }^{2+}$. [b] Values corresponding to a $S_{1}$ saddle point structure (no $S_{2}$ minimum for $4^{4+}$; see Figure 3 ). 
Remarkably, the quantum yield for the photo-induced ringopening isomerization of $\mathbf{4}^{{ }^{4+}}$ is $\Phi_{c / 0}=1.4 \cdot 10^{-4},{ }^{[7]}$ determined by actinometry $(\lambda=480 \mathrm{~nm})$ using 2,7-di-tert-butyl-trans-10b,10cdimethyl-10b,10c-dihydropyrene as standard, showing a significant 300-fold decrease compared to the mono and bispyridinium DHPs. Such a drastic change of photoswitching efficiency necessarily coincides with a substantial change of the photochemical pathway. In other words, important modifications in the potential energy landscape must occur such that the formation of $\mathrm{CPD}^{*}$ is impeded in $\mathbf{4 c}^{\mathbf{4}^{+}}$. In order to rationalize this unexpected behavior, TD-DFT calculations were performed to investigate the initial photochemical pathway.

The first remarkable feature concerns the change of nature of the two lowest excited states $S_{1}$ and $S_{2}$ between the bispyridinium and tetrapyridinium DHPs (Table 5). In fact, the $S_{1}$ and $S_{2}$ excited states of $4_{c}{ }^{4+}$ correlate directly with the $S_{1} L_{b}$ and $S_{2} L_{a}$ states of the reference $1_{c}$, as shown by the direct comparison of the natural transition orbitals (NTOs) involved in the $S_{1}$ and $S_{2}$ states of these two DHPs. A consequence is that the associated potential energy surfaces present a similar topology between $1 \mathrm{c}$ and $4 \mathrm{c}^{4+}$, but very different from that of $3_{c}{ }^{2+}$. In the bispyridinium DHP $3_{c}{ }^{2+}$, the $S_{1}$ potential energy surface presents a minimum corresponding to $\mathrm{CPD}^{*}$ and the relaxation on this surface leads the system directly to this minimum without any surface crossing involved. ${ }^{[4]}$ In the tetrapyridinium DHP $4_{c}{ }^{4+}$, the $S_{1}$ minimum does not correspond to $\mathrm{CPD}^{*}$ but to $\mathrm{DHP}^{*}$ (i.e., the $\mathrm{L}_{b}$ state minimum of similar structure as ground-state DHP), as illustrated by the excited-state geometrical distortions (Table 4). The CPD* structure is associated with a noticeable elongation of the transannular $\mathrm{C}-\mathrm{C}$ bond by $0.07 \AA$ and an increase of the non-planarity of the DHP core of $13.4^{\circ}$ with respect to the ground-state structure. CPD* is thus diabatically linked to the $S_{2} L_{a}$ state and is reached from $S_{2}$ through a peaked $S_{2} / S_{1}$ conical intersection, as shown in Figure 3 . This is similar to what was found in the reference DHP $1_{c} .^{[6,12]}$ The main difference is that CPD* is a first-order saddle point on the $S_{1}$ potential energy surface in $\mathbf{4 c}^{\mathbf{4 +}}$, while it is a minimum in $\mathbf{1}$. This is reminiscent of a second-order Jahn-Teller effect, where the derivative coupling between $S_{1}$ and $S_{2}$ is responsible for the negative curvature at CPD*. Thus, CPD* is an unstable structure in the tetrapyridinium DHP $4 \mathrm{c}^{4+}$, and relaxation from CPD* leads preferentially to $\mathrm{DHP}^{*}$ (Figure 3 ). This peculiar potential energy landscape accounts for the remarkably low $\mathrm{DHP} \rightarrow \mathrm{CPD}$ photoswitching efficiency in this compound (even lower than that of the reference DHP $1 \mathrm{c}$ by a factor 40 ).

Table 5. Comparison of the first two electronic transitions in various DHPs in acetonitrile.

\begin{tabular}{|c|c|c|c|c|c|}
\hline System & State & $\begin{array}{l}\text { Excitation } \\
\text { Energy (eV) }\end{array}$ & $\begin{array}{l}\lambda \\
(\mathrm{nm})\end{array}$ & $f^{[a]}$ & $\mathrm{NTOs}^{[\mathrm{b}]}$ \\
\hline \multirow[t]{2}{*}{ 1c } & $S_{1}\left(A_{u}\right)$ & 2.189 & 567 & 0.003 & \\
\hline & $\mathrm{S}_{2}\left(\mathrm{~B}_{\mathrm{u}}\right)$ & 2.567 & 483 & 0.030 & \\
\hline $3 c^{2+}$ & $S_{1}\left(A_{u}\right)$ & 1.968 & 630 & 0.080 & \\
\hline
\end{tabular}

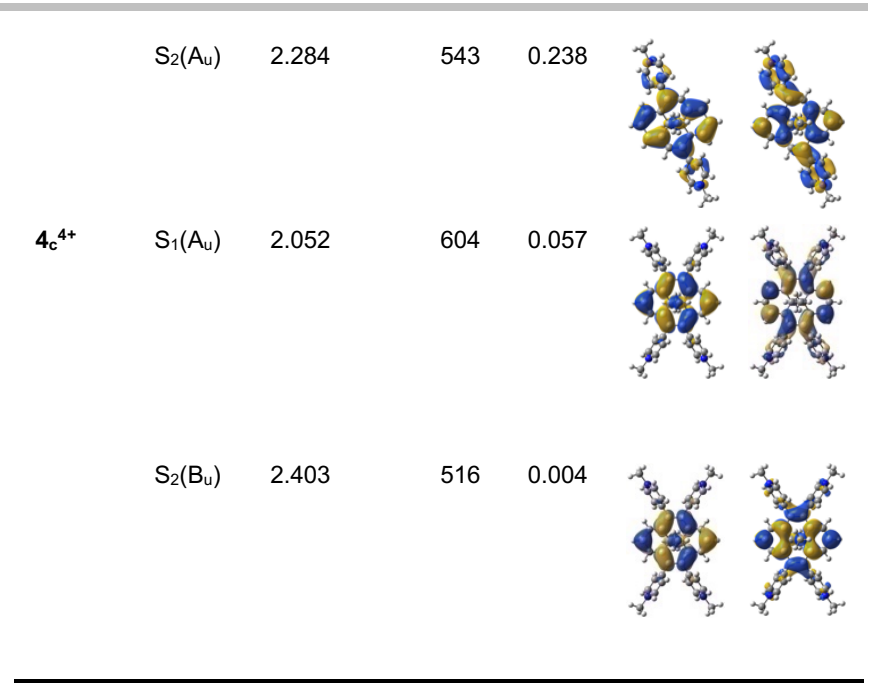

[a] Oscillator strength. [b] Main pair of natural transition orbitals.

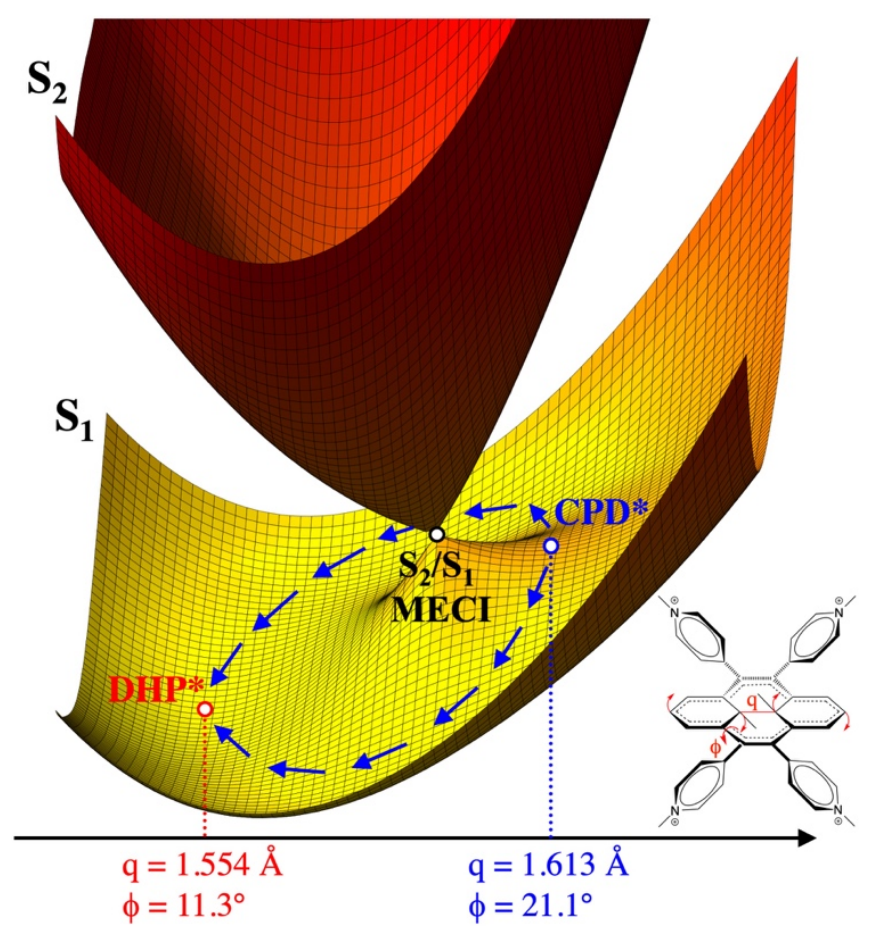

Figure 3. Illustration of the $S_{1}$ and $S_{2}$ potential energy landscape in $4_{c}{ }^{4+}$. DHP* is a minimum on the $S_{1}$ potential energy surface, while $C P^{*}$ is a first-order saddle point diabatically connected to $S_{2}$ via the $S_{2} / S_{1}$ minimum energy conical intersection $(\mathrm{MECl})$. Blue arrows indicate the preferential relaxation from $\mathrm{CPD}^{*}$ to $\mathrm{DHP}^{*}$. The transannular central $\mathrm{C}-\mathrm{C}$ bond length $(\mathrm{q})$ and the average twisting angle between the pyridinium and the DHP core $(\phi)$ are indicated at the DHP* and $\mathrm{CPD}^{*}$ structures.

It is noteworthy that the effect of the four pyridinium units cancel out, while the dissymmetry brought by one or two pyridinium units alters the excited states in favor of the DHP $\rightarrow$ CPD photoisomerization. In the following, we try to provide some possible explanation for this surprising effect. The first factor that comes to mind is a steric one. As mentioned before, the ground-state structure of $4 c^{4+}$ mainly differs from that of $2 c^{+}$and $3_{c}{ }^{2+}$ by the torsion angle of the pyridinium groups. Because of the proximity and bulkiness of these groups, the decrease of the steric repulsion requires that the pyridinium rotates further relative to the 
quasi-planar DHP core in $\mathbf{4 c}^{4+}$. As a result, the torsion angle $\phi$ increases by $30^{\circ}$ and $22^{\circ}$, with respect to that in $2 \mathrm{c}^{+}$and $3_{\mathrm{c}}{ }^{2+}$. Thus the pyridinium are less conjugated to the DHP moiety and the charge transfer character in the excited states is expected to decrease. If this was the case, one would expect that the excited states of $\mathbf{4}{ }^{4+}$ would resemble those of unsubstituted $\mathbf{1}_{c}$, with weak electronic contribution from the pyridinium groups. Comparing the NTOs associated with the $S_{1}$ and $S_{2}$ excited states of $1_{c}$, and $4_{c}{ }^{4+}$ (Table 5), this scenario seems plausible as almost no charge transfer to the pyridinium is observed in $4_{c}{ }^{4+}$, whereas this charge transfer is pronounced in $3_{c}{ }^{2+}$. Figure 4 represents for $\mathbf{4}^{4+}$ the $S_{1}$ and $S_{2}$ potential energy profiles along the pyridinium torsion angle $\phi$ following a relaxed scan on $\mathrm{S}_{0}$. The representation of the NTOs for these two excited states at the ground-state geometry (i.e., at $\phi=64^{\circ}$ ) and at a $\phi$ angle of $40^{\circ}$ (i.e., similar to the value of $\phi$ for the $S_{0}$ structure of $3_{c^{2+}}$ ) confirms that the charge transfer character increases as the torsion angle decreases. However, the order of the electronic states is unchanged along the torsion coordinate. Similarly, increasing the pyridinium torsion in $3 \mathrm{c}^{2+}$ leads to a decrease of the charge transfer character as expected, but no electronic state crossing is observed (Figure S2 in Supporting Information). In other words, even when inducing the charge transfer between the pyridinium groups and the DHP core upon reducing the torsion angle in $4_{c}{ }^{4+}$, the ${ }^{1} B_{u}$ state remains $S_{2}$. Thus, although the torsion controls the degree of charge transfer, it does not account for the order of the ${ }^{1} A_{u}$ and ${ }^{1} B_{u}$ electronic states.

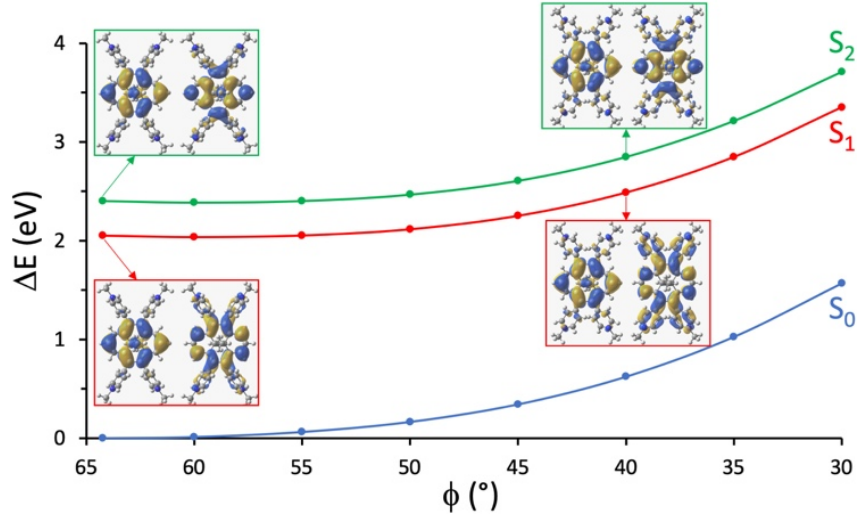

Figure 4. Potential energy profiles of the $S_{0}, S_{1}$ and $S_{2}$ electronic states of $4_{c}{ }^{4+}$ along the pyridinium torsion angle $\phi$. Illustration of the NTOs associated with the electronic transition to $S_{1}$ and $S_{2}$ are shown at two different torsion angles.

In order to explain why the ${ }^{1} \mathrm{~B}_{u}$ photo-isomerizing state is $S_{2}$ in $1_{c}$ and $4 \mathrm{c}^{4+}$, whereas the correlated state becomes $S_{1}$ in $\mathbf{2}^{+}$and $\mathbf{3 c}^{2+}$, we need to consider electronic factors and symmetry consideration. The orbitals involved in the single-excitation transition of the $S_{2}$ state in $\mathbf{1}_{\mathrm{c}}$ and $\mathbf{4}_{\mathrm{c}}{ }^{{ }^{+}}$belong respectively to the $\mathrm{a}_{\mathrm{g}}$ and $\mathrm{b}_{\mathrm{u}}$ irreducible representation of the $C_{2 h}$ point group. These orbitals match with the pair of NTOs associated with the $S_{2}\left(B_{u}\right)$ electronic state shown in Table 5 for these two DHPs. The $a_{g}$ orbital (HOMO) has a bonding character along the $\mathrm{C}-\mathrm{C}$ transannular bond, whereas the $b_{u}$ orbital (LUMO in $\mathbf{1}_{c}$ and LUMO+1 in $\mathbf{4 c}^{\mathbf{4}^{+}}$) has an anti-bonding character for this bond (Figure S3 in Supporting Information). Upon symmetry reduction to the $C_{i}$ point group in $\mathbf{3 c}^{2+}$, these orbitals belong respectively to the $a_{g}$ and $a_{u}$ irreducible representation, meaning that this state now becomes an $A_{u}$ electronic state. Of course, the $S_{1}\left(A_{u}\right)$ state of $1_{c}$ and $4_{c}{ }^{4+}$, which is dominated by an $a_{g} \rightarrow a_{u}$ transition ${ }^{[13]}$ (where $a_{u}$ has a non-bonding character along the $\mathrm{C}-\mathrm{C}$ transannular bond; Figure S3 in Supporting Information), remains an $A_{u}$ state upon symmetry reduction to the $C_{i}$ point group in $3 \mathrm{c}^{2+}$. Thus, the two lowest excited states $S_{1}$ and $S_{2}$ both belong to the same irreducible representation in $3_{c^{2+}}$ (a similar statement is valid for $\mathbf{2}^{+}$for which no symmetry exists). The $S_{1}\left(A_{u}\right)$ and $S_{2}\left(A_{u}\right)$ can therefore mix in $C_{i}$ symmetry. This mixing is clearly happening through orbital mixing. Indeed, the second NTO of the $S_{1}\left(A_{u}\right)$ state in $3_{c}{ }^{2+}$ appears to be a mixture of the second NTOs of the $S_{1}\left(A_{u}\right)$ and $S_{2}\left(B_{u}\right)$ states in the more symmetric $1_{c}$ and $4_{c}{ }^{4+}$ molecules. In other words, this $S_{1}$ state in $3_{c}{ }^{2+}$ correlates with a mixture of the $S_{1}\left(A_{u}\right)$ and $S_{2}\left(B_{u}\right)$ states of $1_{c}$ or $4_{c}{ }^{4+}$. A structural manifestation of this fact is that the excited-state deformations in the $S_{1}$ state of $2_{c}{ }^{+}$ and of $3 c^{2+}$ are lying in between those of the $S_{1}$ and $S_{2}$ states of $1 \mathrm{c}$ and $4 \mathrm{c}^{4+}$ : for example, the increase of the $\phi$ angle with respect to the ground state is about $7^{\circ}$ in the relaxed $S_{1}$ state of $3_{c}{ }^{2+}$, whereas it is respectively $2^{\circ}$ and $10^{\circ}$ in the $S_{1}$ and $S_{2}$ states of $1_{c}$ (Table 4). This is also consistent with the fact that the excited $a_{u}$ LUMO orbital in $3 \mathrm{c}^{2+}$ has a less pronounced anti-bonding character along the central C-C bond than the bu orbital in $4_{c}{ }^{4+}$ (Figure S3 in Supporting Information).

A more delicate question to address concerns the reason for the photo-activity of the $S_{1}$ state in $4_{c}{ }^{4+}$. As explained above, this state correlates with the $S_{1}$ covalent $L_{b}$ state of $1_{c}$, which does not lead to photo-isomerization in the unsubstituted compound. Several reasons could come to mind. The first one is that the $S_{0} \rightarrow S_{1}$ transition in $\mathbf{4 c}^{4+}$ is more efficient upon red light irradiation compared to $1_{c}$, with a ratio of ca. 20 in the oscillator strengths in favor of $\mathbf{4 c}^{\mathbf{4}^{+}}$(Table S3 in Supporting Information). A second argument is that the $S_{1}$ potential energy well is deeper in $\mathbf{1}_{\mathrm{c}}$ compared to the one in $4 c^{4+}$. The energy difference between DHP* and $\mathrm{CPD}^{*}$ reduces from $4.9 \mathrm{kcal} . \mathrm{mol}^{-1}$ in $1_{\mathrm{c}}$ to $3.4 \mathrm{kcal}^{\mathrm{mol}}{ }^{-1}$ in $4_{c}{ }^{4+}$ giving the latter system more chances to escape from the unreactive $\mathrm{DHP}^{*}$ excited-state intermediate to the photoreactive $\mathrm{CPD}^{*}$ excited-state species. Another reason could be that this state may undergo a more efficient radiationless deactivation back to the initial ground state in $\mathbf{1}_{\mathrm{c}}$ than in $\mathbf{4 c}^{{ }^{4+}}$. In other words, the $S_{0} / S_{1}$ conical intersection could be more difficult to access in $4_{c}{ }^{4+}$ than in $1_{c}$ possibly due to the bulkiness of the pyridinium groups. However, this hypothesis cannot be substantiated, as the photophysical nonradiative decay path from $\mathrm{DHP}^{*}$ to $\mathrm{DHP}$ is still not well understood. ${ }^{[6]}$

\section{Conclusion}

We investigated theoretically and experimentally the photoisomerizing capability of a tetrapyridinium-appended derivative $4_{c}{ }^{4+}$ of the dimethyldihydropyrene photochromic system $1 \mathrm{c}$. The structures and electronic properties of the ground and lowest electronic excited states of $\mathbf{4 c}^{\mathbf{4}^{+}}$are described. This derivative displays a surprisingly low quantum yield for the photo-induced ring-opening isomerization from DHP to CPD with a 300 -fold decrease relative to that of the mono $2^{+}$and bispyridinium $3 c^{2+}$ DHPs.

The comparison of the electronic structures of the first two singlet excited states $S_{1}$ and $S_{2}$ between these pyridinium-appended derivatives and the reference dimethyldihydropyrene compound shows that $1_{c}$ and $4_{c}{ }^{4+}$ share the same set of excited states, with both $S_{1}$ and $S_{2}$ being photo-isomerizing states in $4 c^{4+}$, albeit with a very low efficiency. The analysis of the topological features of 
the $S_{1}$ and $S_{2}$ potential energy surfaces predict the transient excited-state precursor for the CPD formation $\left(\mathrm{CPD}^{*}\right)$ to be unstable and short-lived in $4^{4+}$ because it corresponds to a saddle-point structure on the $S_{1}$ potential energy surface in the vicinity of an easily accessible $S_{1} / S_{2}$ conical intersection. Thus, after an efficient $S_{2} \rightarrow S_{1}$ nonradiative decay to $C P D^{*}$, the system will relax preferentially to the lower $S_{1} D_{H} P^{*}$ minimum, an unreactive excited-state species. However, $\mathbf{4 c}^{4+}$ can still be isomerized into $4^{4+}$ by direct excitation to $S_{1}$ with red light irradiation, something not achievable in $\mathbf{1}_{\mathrm{c}}$. This could possibly be explained by a more efficient $S_{0} \rightarrow S_{1}$ transition and a shallower $\mathrm{DHP}^{*}$ potential energy well, allowing the system to reach the reactive $\mathrm{CPD}^{*}$ region.

In the mono $2 \mathrm{c}^{+}$and bispyridinium $3 \mathrm{c}^{2+}$ DHPs, the situation is different as the reduction of symmetry allows for orbital and state mixing. As a result, the $S_{1}$ state in these systems can be viewed as a mixture of the $S_{1}\left(A_{u}\right)$ and $S_{2}\left(B_{u}\right)$ states of $1_{c}$ or $4_{c}{ }^{4+}$, producing the CPD* directly on the lowest $S_{1}$ excited state. This study thus shows that charge transfer to the pyridinium groups is not the only reason for the increased isomerization efficiency but that symmetry reduction or symmetry breaking also plays a crucial role.

\section{Computational and Experimental Section}

Computational Section. DFT has been used to perform calculations of closed-shell and open-shell species for isolated model systems of the reference compound (1), of the monopyridinium- (2), bispyridinium- (3) and tetrapyridinium-appended (4) DHP derivatives in the ground electronic state. All the model systems were obtained by simply replacing the bulky tert-butyl groups by hydrogen atoms in order to reduce the computational cost. Geometry optimizations were carried out for the closed-ring and open-ring isomers of all the compounds and for the transition states connecting them. These transition states correspond to open-shell singlet (biradical) species and were computed using broken-symmetry DFT calculations. To account for the spin contamination, spin-projected energies were computed with the approximated spin-correction procedure proposed by Yamaguchi and coworkers. ${ }^{[14]}$ All these geometry optimizations were performed with the B3LYP functional. ${ }^{[15]}$ Excited-state geometry optimizations and potential energy profile calculations were performed at the TD-DFT level using the CAM-B3LYP functional. ${ }^{[16]} \mathrm{DHP}^{*}$ and $C P D^{*}$ were obtained by geometry optimization of the $S_{1}\left(A_{u}\right)$ and $S_{2}\left(B_{u}\right)$ states, respectively. The schematic representation of the $S_{1}$ and $S_{2}$ potential energy surfaces shown in Figure 3 results from the energy and structural data along these geometry optimizations and from the numerical frequency calculations performed at these stationary points. The absorption spectrum of $\mathbf{4 c}^{\mathbf{4}^{+}}$was computed in acetonitrile using the polarizable continuum model (PCM). Natural transition orbitals (NTOs) ${ }^{[11]}$ for relevant electronic transitions were generated in order to analyze the nature of the excited states involved. The triple- $\zeta$ quality basis set including polarization functions $6-311 \mathrm{G}(\mathrm{d}, \mathrm{p})$ was used throughout. ${ }^{[17]}$ All calculations were performed with Gaussian $09^{[18]}$ using the default convergence criteria for energies and gradients along with an ultrafine integration grid. All the optimized Cartesian coordinates and energies are collected in Table S2 in Supporting Information.

Experimental Section. The synthesis of the tetrapyridinium-appended dimethyldihydropyrene $\mathbf{4 c}^{{ }^{4+}}$ is described in ref. [7]. Irradiation experiments in solution were performed under inert atmosphere. The visible irradiations for making the isomerization of the closed-ring (DHP) isomers to their corresponding open-ring (CPD) forms were carried out with a Xe-Hg lamp $(500 \mathrm{~W})$, using a $\lambda>630 \mathrm{~nm}$ cut-off filter and the samples were placed in an ice/water bath in order to limit the reverse thermal reaction. Single crystal X-ray diffraction data have been collected at $200 \mathrm{~K}$ on a Bruker AXS Enraf-Nonius Kappa CCD diffractometer using the Mo-K $\mathrm{K}_{\alpha}$ radiation from an Incoatec microsource. Intensity data have been collected for Lorentzpolarization effects and absorption with the Olex ${ }^{2}$ software. Structures solutions and refinements have been performed with 0 lex ${ }^{2}$ by direct methods. All non-hydrogen atoms have been refined by full matrix least squares with anisotropic thermal parameters. Hydrogen atoms have been introduced at calculated positions as riding atoms. Crystallographic structures have been drawn using the Mercury 3.1 software. A summary of the data collection and structure refinements is provided in Table $\mathrm{S} 1$ in Supporting Information. Absorption spectra of $\left[\mathbf{4}_{\mathbf{c}}\left(\mathrm{PF}_{6}\right)_{4}\right]$ and of its openring isomer were recorded in acetonitrile solution using a Varian Cary 50 Scan UV-visible spectrophotometer equipped with a temperature controller unit. Absorption measurements were carried out in quartz cells with optical path length of $1 \mathrm{~cm}$.

\section{Acknowledgements}

This work was granted access to the HPC resources of CALMIP supercomputing center under the allocation 2019-[12158]. The authors acknowledge the financial support from Agence Nationale de la Recherche through grant no. ANR-18-CE29-0012 (Photochromics project). The NanoBio-ICMG platforms (FR 2607) are acknowledged for their support.

Keywords: Computational Chemistry - Conical Intersection • Excited States $\cdot$ Photochromism • Photo-isomerization

[1] a) V. Balzani, A. Credi, M. Venturi in Molecular Devices and Machines, Wiley-VCH, Weinheim, 2008; b) B. L. Feringa, W. R. Browne, Molecular Switches, Volume 2: Second, Completely Revised and Enlarged Edition, Wiley-VCH Verlag GmbH \& Co. KGaA, 2011.

[2] a) R. H. Mitchell, Eur. J. Org. Chem. 1999, 2695-2703; b) R. H. Mitchell, T. R. Ward, Y. Wang, P. W. Dibble, J. Am. Chem. Soc. 1999, 121, 26012602; c) R. S. Murphy, Y. Chen, T. R. Ward, R. H. Mitchell, C. Bohne, Chem. Commun. 1999, 2097-2098; d) R. H. Mitchell, Z. Brkic, V. A. Sauro, D. J. Berg, J. Am. Chem. Soc. 2003, 125, 7581-7585; e) S. D. Straight, J. Andréasson, G. Kodis, S. Bandyopadhyay, R. H. Mitchell, T. A. Moore, A. L. Moore, D. Gust, J. Am. Chem. Soc. 2005, 127, 94039409; f) R. H. Mitchell, C. Bohne, Y. Wang, S. Bandyopadhyay, C. B. Wozniak, J. Org. Chem. 2006, 71, 327-336; g) R. H. Mitchell, C. Bohne, S. G. Robinson, Y. Yang, J. Org. Chem. 2007, 72, 7939-7946; h) S. Muratsugu, S. Kume, H. Nishihara, J. Am. Chem. Soc. 2008, 130, 72047205; i) N. Vilà, G. Royal, F. Loiseau, A. Deronzier, Inorg. Chem. 2011, 50, 10581-10591; j) S. Muratsugu, M. Kishida, R. Sakamoto, H. Nishihara, Chem. Eur. J. 2013, 19, 17314-17327; k) D. Roldan, V. Kaliginedi, S. Cobo, V. Kolivoska, C. Bucher, W. Hong, G. Royal, T. Wandlowski, J. Am. Chem. Soc. 2013, 135, 5974-5977; I) M. Kishida, T. Kusamoto, H. Nishihara, J. Am. Chem. Soc. 2014, 136, 4809-4812; m) S. Cobo, F. Lafolet, E. Saint-Aman, C. Philouze, C. Bucher, S. Silvi, A. Credi, G. Royal, Chem. Commun. 2015, 51, 13886-13889; n) A. Bakkar, S. Cobo, F. Lafolet, D. Roldan, E. Saint-Aman, G. Royal, J. Mater. C 2016, 4, 1139-1143; o) Y. Garmshausen, K. Klaue, S. Hecht, ChemPlusChem 2017, 82, 1025-1029; p) K. Klaue, Y. Garmshausen, S. Hecht, Angew. Chem. Int. Ed. 2018, 57, 1414-1417; q) A. Bakkar, F. Lafolet, D. Roldan, E. Puyoo, D. Jouvenot, G. Royal, E. Saint-Aman, S. Cobo, Nanoscale 2018, 10, 5436-5441; r) G. P. Zhang, Y. Q. Mu, J. M. Zhao, H. Huang, G. C. Hu, Z. L. Li, C. K. Wang, Physica E 2019, 109, 15; s) Q. K. Wang, G. Ligorio, R. Schlesinger, V. Diez-Cabanes, D. Cornil, Y. Garmshausen, S. Hecht, J. Cornil, E. J. W. Kratochvil, N. Koch, Adv. Mater. Interfaces 2019, 6, 1900211; t) M. Jacquet, L. M. Uriarte, F. Lafolet, M. Boggio-Pasqua, M. Sliwa, F. Loiseau, E. Saint-Aman, S. Cobo, G. Royal, J. Phys. Chem. Lett. 2020, 11, 2682-2688.

[3] H. Bouas-Laurent, H. Dürr, Pure Appl. Chem. 2001, 73, 639-665.

[4] D. Roldan, S. Cobo, F. Lafolet, N. Vilà, C. Bochot, C. Bucher, E. SaintAman, M. Boggio-Pasqua, M. Garavelli, G. Royal, Chem. Eur. J. 2015, $21,455-467$. 
[5] M. A. L. Sheepwash, R. H. Mitchell, C. Bohne, J. Am. Chem. Soc. 2002, 124, 4693-4700.

[6] M. Boggio-Pasqua, M. J. Bearpark, M. A. Robb, J. Org. Chem. 2007, 72, 4497-4503.

[7] A. Bakkar, F. Lafolet, M. Boggio-Pasqua, D. Jouvenot, E. Saint-Aman, S. Cobo, Chem. Commun. 2017, 53, 9360-9363.

[8] M. Jacquet, F. Lafolet, S. Cobo, F. Loiseau, A. Bakkar, M. BoggioPasqua, E. Saint-Aman, G. Royal, Inorg. Chem. 2017, 56, 4357-4368.

[9] A. Bakkar, S. Cobo, F. Lafolet, M. Boggio-Pasqua, G. Royal, E. SaintAman, Dalton Trans. 2016, 45, 16453-16462.

[10] R. V. Williams, W. D. Edwards, R. H. Mitchell, S. G. Robinson, J. Am Chem. Soc. 2005, 127, 16207-16214.

[11] R. L. Martin, J. Chem. Phys. 2003, 118, 4775-4777.

[12] M. Boggio-Pasqua, M. Garavelli, J. Phys. Chem. A 2015, 119, 6024-6032.

[13] R. Sarkar, M.-C. Heitz, G. Royal, M. Boggio-Pasqua, J. Phys. Chem. A 2020, 124, 1567-1579.

[14] a) K. Yamaguchi, F. Jensen, A. Dorigo, K. N. Houk, Chem. Phys. Lett. 1988, 149, 537-542; b) S. Yamanaka, T. Kawakami, H. Nagao, K. Yamaguchi, Chem. Phys. Lett. 1994, 231, 25-33.

[15] A. D. Becke, J. Chem. Phys. 1993, 98, 5648-5652.

[16] T. Yanai, D. P. Tew, N. C. Handy, Chem. Phys. Lett. 2004, 393, 51-57.

[17] R. Krishnan, J. S. Binkley, R. Seeger, J. A. Pople, J. Chem. Phys. 1980, 72, 650-654.

[18] M. J. Frisch, G. W. Trucks, H. B. Schlegel, G. E. Scuseria, M. A. Robb, J. R. Cheeseman, G. Scalmani, V. Barone, G. A. Petersson, H. Nakatsuji, X. Li, M. Caricato, A. Marenich, J. Bloino, B. G. Janesko, R. Gomperts, B. Mennucci, H. P. Hratchian, J. V. Ortiz, A. F. Izmaylov, J. L. Sonnenberg, D. Williams-Young, F. Ding, F. Lipparini, F. Egidi, J. Goings, B. Peng, A. Petrone, T. Henderson, D. Ranasinghe, V. G. Zakrzewski, J Gao, N. Rega, G. Zheng, W. Liang, M. Hada, M. Ehara, K. Toyota, R. Fukuda, J. Hasegawa, M. Ishida, T. Nakajima, Y. Honda, O. Kitao, H. Nakai, T. Vreven, K. Throssell, J. A. Montgomery, Jr., J. E. Peralta, F. Ogliaro, M. Bearpark, J. J. Heyd, E. Brothers, K. N. Kudin, V. N. Staroverov, T. Keith, R. Kobayashi, J. Normand, K. Raghavachari, A Rendell, J. C. Burant, S. S. lyengar, J. Tomasi, M. Cossi, J. M. Millam M. Klene, C. Adamo, R. Cammi, J. W. Ochterski, R. L. Martin, K Morokuma, O. Farkas, J. B. Foresman, and D. J. Fox, Gaussian 09 , Revision D.01, Gaussian, Inc., Wallingford CT, 2009. 


\section{Entry for the Table of Contents}

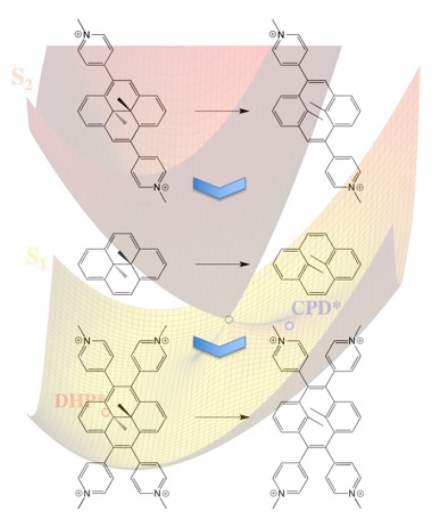

While substitution with one or two pyridinium electron-withdrawing groups improves the dimethyldihydropyrene (DHP) photoswitchability, the use of four pyridinium groups has a surprising detrimental effect. We report a theoretical study comparing the excited-state properties of these DHP compounds. A rationale for this unexpected behavior is provided based on topological features of the potential energy surfaces and symmetry arguments.

Institute and/or researcher Twitter usernames: @LCPQ_UMR5626 